\title{
New SLICOT Routines Based on Structured Eigensolvers*
}

\author{
Vasile Sima $^{1}$, Peter Benner ${ }^{2}$, and Daniel Kressner ${ }^{3}$
}

\begin{abstract}
The latest developments of the SLICOT Library based on structure preserving algorithms for solving eigenproblems with Hamiltonian matrices and skewHamiltonian/Hamiltonian matrix pencils are discussed. An important application benefiting from these advances is the computation of $\mathscr{L}_{\infty}$-norms of linear time-invariant multivariable systems. It is shown that this approach delivers accurate results and it is much faster than the state-of-the-art tools.
\end{abstract}

\section{INTRODUCTION}

The last years have recorded advances in the computation of spectra and selected eigenspaces for structured matrices or matrix pencils. The main research efforts have been directed to algorithms and associated software which are able to exploit the specific structure, in order to improve the reliability, accuracy and efficiency of the calculations. Important structures for control theory and applications are Hamiltonian matrices and skew-Hamiltonian/Hamiltonian matrix pencils. Theory and algorithmic details are presented, e.g., in [1], [2], [3], [4] and the references therein.

Based on these advances, the Subroutine Library In COntrol Theory-SLICOT-has been recently expanded with algorithms for computations with (skew-)Hamiltonian matrices and skew-Hamiltonian/Hamiltonian matrix pencils. Specifically, an important SLICOT update took place in June 2009, when many new subroutines, mainly standardized versions of the HAPACK routines (see http: //www.tu-chemnitz.de/mathematik/hapack/) for (skew-)Hamiltonian eigenvalue problems, have been added. (Related papers can be found at http://www . tu-chemnitz.de/mathematik/hapack/pub/.)

The latest important update of the SLICOT Library took place in November 2010, when 29 new routines related to solving eigenproblems for skew-Hamiltonian/Hamiltonian matrix pencils have been added. Spectra and bases of stable invariant or deflating subspaces can be computed for such structured matrices or matrix pencils. Several applications benefit from the structure exploitation, including the calculation of $\mathscr{L}_{\infty^{-}}$or $\mathscr{H}_{\infty}$-norms of linear time-invariant multivariable systems and solution of algebraic matrix Riccati equations. The SLICOT Library contents, the associated MATLAB ${ }^{\circledR}$ (and Scilab) tools, and their

\footnotetext{
*This work was supported in part by the Deutsche Forschungsgemeinschaft under grant BE-2174/6-1 and by The MathWorks, Inc.

${ }^{1} \mathrm{~V}$. Sima is with Advanced Research Department, National Institute for Research and Development in Informatics, 011455 Bucharest, Romania vsimalici.ro

${ }^{2} \mathrm{P}$. Benner is with the Max Planck Institute for Dynamics of Complex Technical Systems, 39106 Magdeburg, Germany benner@mpi-magdeburg.mpg. de

${ }^{3}$ D. Kressner is with the École Polytechnique Fédérale de Lausanne EPFL, 1015 Lausanne, Switzerland daniel.kressner@epfl.ch
}

performance have been presented, e.g., in [5], [6], [7], [8] Recent publications concerned with implementation issues and numerical results include [9], [10], [11], [12].

The new routines and tools have been already used in applications such as those mentioned above. A new routine, AB13HD, for the computation of the $\mathscr{L}_{\infty}$-norm of standard or descriptor systems has been designed, based on the new, structure preserving algorithms. This routine is an improvement of $A B 13 D D$, described in [13], where the very good performance for continuous-time standard systems has been shown. AB13DD then used the square-reduced method for Hamiltonian matrices (see [14] and the references there). Later, AB13DD has been improved by using the HAPACK approach (including suitable Hamitonian matrix scaling) for the same class of systems. The routine AB13HD adds the structure preserving approach for discrete-time and descriptor systems. The supporting theory and its performance have been summarized in [15]. This new routine proved equally accurate, but much faster than the state-of-the-art MATLAB function norm from the MATLAB Control System Toolbox [16], called via the command norm (sys, inf), where sys represents a linear time-invariant system.

This paper discusses the main functionality added by the latest developments of the SLICOT Library, and summarizes the numerical experience in using the new software on a large collection of dynamical systems [17].

\section{EXPLOITING HAMILTONIAN-LIKE STRUCTURES}

A matrix pencil $\mathscr{N}-\lambda \mathscr{M}$ is Hamiltonian if $\mathscr{N} \mathscr{J} \mathscr{M}^{H}=$ $-\mathscr{M} \mathscr{J} \mathscr{N}^{H}$, and it is symplectic if $\mathscr{N} \mathscr{J} \mathscr{N}^{H}=\mathscr{M} \mathscr{J} \mathscr{M}^{H}$, where

$$
\mathscr{J}:=\left[\begin{array}{cc}
0 & I_{n} \\
-I_{n} & 0
\end{array}\right], \quad \mathscr{J}^{T}=-\mathscr{J}=\mathscr{J}^{-1},
$$

the superscripts $H$ and $T$ denote the conjugate-transpose and transpose, respectively, and $I_{n}$ denotes the identity matrix of order $n$. If $\mathscr{M}=I_{2 n}$, the usual definitions for Hamiltonian and symplectic matrices are obtained; for instance, $\mathscr{N}$ is Hamiltonian if $(\mathscr{N} \mathscr{J})^{H}=\mathscr{N} \mathscr{J}$, and it is skew-Hamiltonian if $(\mathscr{N} \mathscr{J})^{H}=-\mathscr{N} \mathscr{J}$. A matrix pencil $\lambda \mathscr{M}-\mathscr{N}$ is skewHamiltonian/Hamiltonian if $\mathscr{M}$ is skew-Hamiltonian, and $\mathscr{N}$ is Hamiltonian. These pencils have spectra which are symmetric with respect to the imaginary axis. In the sequel, the pencils $\lambda \mathscr{M}-\mathscr{N}$ will be represented in the numerically better form $\alpha \mathscr{M}-\beta \mathscr{N}$, with $\lambda=\alpha / \beta$ (possibly $\infty$ ). For convenience, the real case only will be considered, although the complex case is also covered in SLICOT.

The computations described below are essentially performed using orthogonal and orthogonal symplectic matrices, 
which confer the algorithms a very good numerical behaviour, making them backward stable and structure preserving.

\section{A. Hamiltonian Matrices}

One of the main functions added to the SLICOT Libraryimplemented in the subroutine MBO3XD-is to compute the eigenvalues of a Hamiltonian matrix

$$
\mathscr{H}=\left[\begin{array}{cc}
A & G \\
Q & -A^{T}
\end{array}\right], \quad G=G^{T}, Q=Q^{T},
$$

where $A, G$, and $Q$ are real $n \times n$ matrices.

Due to the structure of $\mathscr{H}$, the eigenvalues appear in pairs $(\lambda,-\lambda)$. The eigenvalues are computed using an algorithm based on the symplectic URV decomposition and the periodic Schur decomposition, as described in [2],

$$
U^{T} \mathscr{H} V=\left[\begin{array}{cc}
T & W \\
0 & -S^{T}
\end{array}\right],
$$

where $U$ and $V$ are $2 n \times 2 n$ orthogonal symplectic matrices, $S$ is in real Schur form and $T$ is upper triangular. Details on the eigenvalue computation using (2) are given in [2]. The algorithm is backward stable and preserves the eigenvalue pairings in finite precision arithmetic. This is not true for the standard QR algorithm, as implemented in the MATLAB function eig. Optionally, a symplectic balancing transformation to improve the conditioning of eigenvalues is computed (using the SLICOT Library routine MB04DD). In this case, the matrix $\mathscr{H}$ in the decomposition (2) must be replaced by the balanced matrix.

Another SLICOT Library routine, MB03ZD, can be used to compute invariant subspaces of $\mathscr{H}$ from the output of the routine MB03XD.

The routine MB03XP computes the product Schur decomposition and eigenvalues of a matrix product $A B$, with $A$ upper Hessenberg and $B$ upper triangular, without evaluating any part of the product. Specifically, the matrices $Q$ and $Z$ are computed, so that

$$
Q^{T} A Z=S, \quad Z^{T} B Q=T,
$$

where $S$ is in real Schur form, and $T$ is upper triangular.

\section{B. Skew-Hamiltonian/Hamiltonian Matrix Pencils}

One of the main functions added to the SLICOT Library-implemented in the subroutine MBO4BD-is to compute the eigenvalues of a real $2 m \times 2 m$ skewHamiltonian/Hamiltonian pencil $\alpha \mathscr{S}-\beta \mathscr{H}$, with

$$
\mathscr{S}=\left[\begin{array}{cc}
A & D \\
E & A^{T}
\end{array}\right], \quad \mathscr{H}=\left[\begin{array}{cc}
C & V \\
W & -C^{T}
\end{array}\right],
$$

and $D^{T}=-D, E^{T}=-E, V^{T}=V, W^{T}=W$. Optionally, decompositions of $\mathscr{S}$ and $\mathscr{H}$ are computed via orthogonal transformations $\mathscr{Q}_{1}$ and $\mathscr{Q}_{2}$, as follows:

$$
\begin{aligned}
\mathscr{Q}_{1}^{T} \mathscr{S} \mathscr{J} \mathscr{Q}_{1} \mathscr{J}^{T} & =\left[\begin{array}{cc}
\widetilde{A} & \widetilde{D} \\
0 & \widetilde{A}^{T}
\end{array}\right], \\
\mathscr{J}^{T} \mathscr{Q}_{2}^{T} \mathscr{J} \mathscr{S} \mathscr{Q}_{2} & =\left[\begin{array}{cc}
\widetilde{B} & \widetilde{F} \\
0 & \widetilde{B}^{T}
\end{array}\right], \\
\mathscr{Q}_{1}^{T} \mathscr{H} \mathscr{Q}_{2} & =\left[\begin{array}{cc}
\widetilde{C}_{1} & \widetilde{V} \\
0 & \widetilde{C}_{2}^{T}
\end{array}\right],
\end{aligned}
$$

where $\widetilde{A}, \widetilde{B}, \widetilde{C}_{1}$ are upper triangular, $\widetilde{C}_{2}$ is upper quasitriangular and $\widetilde{D}$ and $\widetilde{F}$ are skew-symmetric. Optionally, the orthogonal transformation matrices $\mathscr{Q}_{1}$ and $\mathscr{Q}_{2}$ are computed. Theory and algorithms are given in [4].

A similar function, implemented in the subroutine MB 04AD, computes the eigenvalues and the generalized symplectic URV decomposition for a real $2 m \times 2 m$ factored pencil $\alpha \mathscr{T} \mathscr{Z}-\beta \mathscr{H}, \mathscr{T}:=\mathscr{J} \mathscr{Z}^{T} \mathscr{J}^{T}$, i.e., it determines orthogonal matrices $\mathscr{Q}_{1}, \mathscr{Q}_{2}$ and orthogonal symplectic matrices $\mathscr{U}_{1}, \mathscr{U}_{2}$ such that,

$$
\begin{aligned}
\mathscr{Q}_{1}^{T} \mathscr{T} \mathscr{U}_{1} & =\left[\begin{array}{cc}
T_{11} & T_{12} \\
0 & T_{22}
\end{array}\right], \\
\mathscr{U}_{2}^{T} \mathscr{Z} \mathscr{Q}_{2} & =\left[\begin{array}{cc}
Z_{11} & Z_{12} \\
0 & Z_{22}
\end{array}\right], \\
\mathscr{Q}_{1}^{T} \mathscr{H} \mathscr{Q}_{2} & =\left[\begin{array}{cc}
H_{11} & H_{12} \\
0 & H_{22}
\end{array}\right],
\end{aligned}
$$

where $T_{11}, T_{22}^{T}, Z_{11}, Z_{22}^{T}, H_{11}$ are upper triangular and $H_{22}^{T}$ is upper quasi-triangular. (See [4] for theory and algorithmic details.) The matrices $\mathscr{U}_{i}$ are each represented by two arrays containing the submatrices $U_{i 1}$ and $U_{i 2}, i=1,2$, such that

$$
\mathscr{U}_{1}=\left[\begin{array}{cc}
U_{11} & U_{12} \\
-U_{12} & U_{11}
\end{array}\right], \quad \mathscr{U}_{2}=\left[\begin{array}{cc}
U_{21} & U_{22} \\
-U_{22} & U_{21}
\end{array}\right] .
$$

Another pair of functions, implemented in the subroutines MBO3LD and MBO3FD, compute the stable right deflating subspace of the pencil (3) or its factored variant.

These main "solvers" call several auxiliary routines, performing low-level functions. The following two routines are of great importance.

MB03BD computes the eigenvalues of the generalized formal matrix product $A=A_{1}^{s_{1}} A_{2}^{s_{2}} \cdots A_{k}^{s_{k}}$, where $s_{j}=1$ or $s_{j}=-1, j=1, \ldots, k, A_{h}$ is upper Hessenberg and $A_{i}, i \neq h$, is upper triangular (upper Hessenberg/triangular form), using a double-shift version of the periodic QZ algorithm [18], [19]. In addition, $A$ may be reduced to periodic Schur form: $A_{h}$ is upper quasi-triangular and all the other factors $A_{i}$ are upper triangular. Optionally, the $2 \times 2$ triangular matrices corresponding to $2 \times 2$ diagonal blocks in $A_{h}$ are reduced so that their product is a $2 \times 2$ diagonal matrix. Optionally, the orthogonal factors $Q_{j}$ used, $j=1, \ldots, k$, are accumulated.

MB03KD reorders the eigenvalues of the generalized formal matrix product [20], [21] (with inverse order than in $\mathrm{MB}$ 03BD)

$$
\left(T_{22}^{(K)}\right)^{s_{K}}\left(T_{22}^{(K-1)}\right)^{s_{K-1}} \cdots\left(T_{22}^{(1)}\right)^{s_{1}}
$$

in the generalized periodic real Schur form,

$$
T_{k}=\left[\begin{array}{ccc}
T_{11}^{(k)} & T_{12}^{(k)} & T_{13}^{(k)} \\
0 & T_{22}^{(k)} & T_{23}^{(k)} \\
0 & 0 & T_{33}^{(k)}
\end{array}\right], \quad k=1, \ldots, K,
$$

such that the $m$ selected eigenvalues pointed to by a logical vector select end up in the leading part of the matrix sequence $T_{22}^{(k)}$, where

- the matrices $T_{k}$ are $n_{k+1} \times n_{k}$, if $s_{k}=1$, or $n_{k} \times n_{k+1}$, if $s_{k}=-1$, 
- the submatrices $T_{11}^{(k)}$ are $\widetilde{n}_{k+1} \times \widetilde{n}_{k}$, if $s_{k}=1$, or $\widetilde{n}_{k} \times$ $\widetilde{n}_{k+1}$, if $s_{k}=-1$, and contain dimension-induced infinite eigenvalues,

- the submatrices $T_{22}^{(k)}$ are $\widehat{n} \times \widehat{n}$ and contain core eigenvalues, which are generically neither zero nor infinite,

- the submatrices $T_{33}^{(k)}$ contain dimension-induced zero eigenvalues.

Given that $n_{k}=n_{k+1}$ for all $k$ where $s_{k}=-1$, and the $T_{11}^{(k)}$ are void, then the first $m$ columns of the updated orthogonal transformation matrix sequence $Q_{1}, \ldots, Q_{K}$ span a periodic deflating subspace corresponding to the same eigenvalues.

\section{Application: Computation of the $\mathscr{L}_{\infty}$-norm}

The computation of $\mathscr{L}_{\infty}$ - or $\mathscr{H}_{\infty}$-norms of linear timeinvariant multivariable systems is of fundamental importance for robust control analysis and design. Evaluation of these norms is relatively expensive, especially for systems with large order. Some applications require numerous such evaluations. Therefore, besides reliability and accuracy, computational efficiency is essential. Consider a linear time-invariant generalized system, described by its state-space matrices and the associated transfer-function matrix

$$
G(\lambda)=C(\lambda E-A)^{-1} B+D,
$$

where $A, E \in \mathbb{R}^{n \times n}, B \in \mathbb{R}^{n \times m}, C \in \mathbb{R}^{p \times n}$, and $D \in \mathbb{R}^{p \times m}$, and $\lambda$ is a complex variable replacing the Laplace variable, $s$, for a continuous-time system, and the $Z$-transform variable, $z$, for a discrete-time system. In the standard case, the matrix $E$ is identity of order $n$, but in the descriptor case, $E$ can be singular. Briefly speaking, the $\mathscr{L}_{\infty}$-norm for (4) is defined as the peak gain of the frequency response of $G(\lambda)$. This is finite if the system is proper and the matrix pencil $(A, E)$ has no eigenvalue on the imaginary axis, for a continuoustime system, or on the unit circle, for a discrete-time system. Assuming that the system is stable, the $\mathscr{L}_{\infty}$-norm, also then called $\mathscr{H}_{\infty}$-norm, can be expressed by the least upper bound,

$$
\begin{aligned}
\|G\|_{\infty} & :=\sup _{\mathfrak{R}(s)=0} \sigma_{\max }(G(s)), \\
\|G\|_{\infty} & :=\sup _{\|z\|_{2}=1} \sigma_{\max }(G(z)),
\end{aligned}
$$

in the continuous- and discrete-time case, respectively, where $\mathfrak{R}(\cdot)$ denotes the real part of a complex number, and $\sigma_{\text {max }}(\mathscr{M})$ denotes the largest singular value of a matrix $\mathscr{M}$. For brevity, continuous-time systems only will be considered below.

Quadratically convergent algorithms [22], [23] for the computation of these norms use the purely imaginary eigenvalues of a Hamiltonian or symplectic matrix or matrix pencil at each iteration. (Actually, the pencils arising in the continuous-time case can be rewritten to be skewHamiltonian/Hamiltonian.) Specifically, the Hamiltonian matrix involved in an iteration when $E=I_{n}$ can be written as

$$
\mathscr{M}_{\gamma}=\left[\begin{array}{cc}
A-B R^{-1} D^{T} C & -\gamma B R^{-1} B^{T} \\
\gamma C^{T} S^{-1} C & -A^{T}+C^{T} D R^{-1} B^{T}
\end{array}\right],
$$

where $R=D^{T} D-\gamma^{2} I_{m}, S=D D^{T}-\gamma^{2} I_{p}$, and $\gamma$ is a positive scalar updated during the algorithm. The matrix pencil involved in an iteration can be written as

$$
\lambda \mathscr{N}-\mathscr{M}_{\gamma}=\left[\begin{array}{cc|cc}
\lambda E-A & 0 & -B & 0 \\
0 & \lambda E^{T}+A^{T} & 0 & C^{T} \\
\hline-C & 0 & -D & \gamma I_{p} \\
0 & -B^{T} & \gamma I_{m} & -D^{T}
\end{array}\right],
$$

and it can be put into a skew-Hamiltonian/Hamiltonian form after a possible extension (to get an even order), some permutations and scalings (by -1 ). It can be shown that, under some mild conditions, $\gamma$ is a singular value of $G(\mathrm{i} \omega)$ if and only if $i \omega$ is an eigenvalue of $\lambda \mathscr{N}-\mathscr{M}_{\gamma}$. Details are given in [15] and the references therein. But the detection of purely imaginary eigenvalues is a delicate numerical problem if the standard algorithm is used. Numerical examples illustrating this claim are given, e.g., in [15], [24]. The new SLICOT routine for $\mathscr{L}_{\infty}$-norm computation calls the new Hamiltonian and skew-Hamiltonian/Hamiltonian solvers MB03XD and $\mathrm{MB} 04 \mathrm{BD}$, respectively, to evaluate the eigenvalues and decide if or not some of them are on the imaginary axis. The simple purely imaginary eigenvalues can be evaluated without error in the real part by this approach.

\section{NUMERICAL RESULTS}

This section presents some numerical results, based on an improved version of the Fortran routine AB13DD, called $A B 13 H D$, and a corresponding MATLAB MEX-file. The calculations have been performed on a portable Intel Dual Core computer at $2 \mathrm{GHz}$, with $2 \mathrm{~GB}$ RAM, and relative machine precision $\varepsilon \approx 2.22 \times 10^{-16}$, using Windows XP (Service Pack 2) operating system, Intel Visual Fortran 11.1 compiler, MATLAB 7.13.0.564 (R2011b), and optimized LAPACK and BLAS libraries available with MATLAB. Tolerances have been set to $\sqrt{\varepsilon} \approx 10^{-8}$. The balancing (equilibration) option was not activated for the SLICOT calculations.

The tests reported here have been performed for linear systems from the $\mathrm{COMPl}_{e}$ ib collection [17], which contains 124 standard continuous-time examples (with $E=I_{n}$ ), with several variations, giving a total of 168 problems. The matrix $D$ is zero. All but 16 problems (for systems of order larger than 2000, with matrices in sparse format) have been tried.

\section{A. Standard Case}

The first test set is for the standard case, $E=I_{n}$. The SLICOT solver has the ability to call the matrix pencil routine, MB04BD, if forming the Hamiltonian matrix would lead to serious loss of accuracy. But that routine has never been called in this test set. So, all problems have been successfully solved by the Hamiltonian solver, MB03XD.

In this test, 32 examples have poles on the imaginary axis (in most cases, in the origin), and the $\mathscr{L}_{\infty}$-norm was set to infinity by both SLICOT and MATLAB solvers. The SLICOT solver also sets the $\mathscr{L}_{\infty}$-norm to infinity for the examples CSE 1 and CSE2, which have an eigenvalue at about $-6.42 \cdot 10^{-17}$, and $2.14 \cdot 10^{-16}$, respectively, hence closer to the imaginary axis than the relative machine precision, $\varepsilon$. 
TABLE I

NUMBER OF ITERATIONS AND NUMBER OF TEST FREQUENCIES, WHEN DIFFERENT FOR SLICOT AND MATLAB SOLVERS, APPLIED TO COMPL $_{e}$ IB EXAMPLES

\begin{tabular}{|l||c|c|c|c|}
\hline \multirow{2}{*}{ Examples } & \multicolumn{2}{c|}{ Number of iterations } & \multicolumn{2}{c|}{ Number of frequencies } \\
\cline { 2 - 5 } & SLICOT & MATLAB & SLICOT & MATLAB \\
\hline HE6, HE7 & 3 & 2 & 4 & 2 \\
BDT1 & 1 & 1 & 1 & 0 \\
EB3 & 1 & 1 & 1 & 6 \\
EB4 & 1 & 1 & 1 & 20 \\
EB5 & 1 & 1 & 1 & 40 \\
EB6 & 1 & 1 & 4 & 160 \\
TL & 5 & 1 & 25 & 1 \\
CM1, CM2, CM4 & 1 & 1 & 1 & 2 \\
CM5 & 1 & 1 & 2 & 0 \\
\hline
\end{tabular}

(Matrix $A$ is numerically singular.) The MATLAB function norm reported $\mathscr{L}_{\infty}$-norms of the order $10^{16}$ for CSE1, CSE2.

The $\mathscr{L}_{\infty}$-norms computed by the two solvers have been compared. The SLICOT solver found a slightly larger norm than the MATLAB function norm for 55 examples, and an equal norm for 42 examples. To have a global measure, we used the Euclidean norm of the vector of all finite $\mathscr{L}_{\infty}$ norms. The relative difference of the two Euclidean norms (corresponding to the two solvers) was $2.78 \cdot 10^{-10}$, which shows a very good agreement. However, the difference in the execution time was significant. We used the ratios of the CPU (Central Processing Unit) times for the MATLAB solver and the SLICOT solver as a performance measure. The mean value of these ratios for all 152 examples was about 34 , and their minimum value was about 2.4 (for example AC10). We tried to investigate the reasons for this difference in the execution time. One reason is that the latest versions of the MATLAB solver norm always use a pencil approach, to avoid possible numerical difficulties when forming the Hamiltonian matrix. But as it will be shown later for the descriptor case, this is not the main reason. Another reason could be the different convergence speed of the implemented algorithms. To test this hypothesis, the number of iterations and the number of test frequencies used at each iteration have been recorded for both solvers, but they agreed very well, with few exceptions. The cases where they differ are reported in Table I. The reported numbers of test frequencies for each example are their sum for all iterations. Neither of the examples in Table I has a significant contribution to the total CPU time.

The initial test frequencies (before starting the iterative process) are not counted. Their number differ between the two solvers for 14 examples, and are usually smaller for the MATLAB solver, since it starts by simplifying the system realization. Despite these differences, the $\mathscr{L}_{\infty}$-norms computed by the two solvers agreed very well. Usually, a larger number of iterations or of test frequencies led to a slightly higher $\mathscr{L}_{\infty}$-norm.

Fig. 1 shows the bar graph of the number of iterations needed by the SLICOT $\mathscr{L}_{\infty}$-norm solver versus the number of $\mathrm{COMPl}_{e}$ ib examples. The number of examples requiring 0 , $1, \ldots, 5$ iterations was $34,71,23,16,7$, and 1 , respectively.

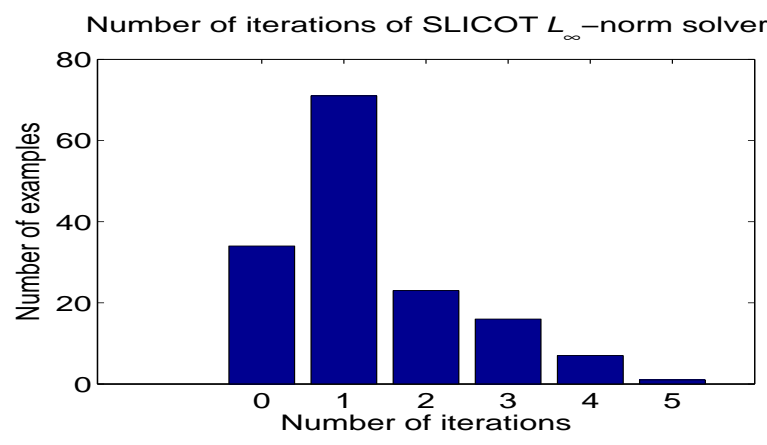

Fig. 1. Bar graph of the number of iterations versus the number of examples from the $\mathrm{COMPl}_{e}$ ib collection.

The first number corresponds to the examples with infinite $\mathscr{L}_{\infty}$-norm.

The number of test frequencies was almost always 2 in each iteration, except for the last, when it was 0 for all examples, except BDT1, EB3-EB5, CM1, CM2, and CM4 with one test frequency, CM3 and CM5 with 2, and EB 6 with 4 test frequencies. For the TL example, the SLICOT solver used $3,16,4,2$, and 0 test frequencies in the five iterations.

The small number of iterations shows the fast convergence of the algorithm. The similar behavior of the two solvers, but the big difference in the execution times, show that the structure preserving SLICOT implementation is very attractive. Differences in implementation details also count.

\section{B. Descriptor Case}

The second test set is for the descriptor case, when the SLICOT skew-Hamiltonian/Hamiltonian solver has been called. To generate descriptor systems with singular matrix $E$ for the $\mathrm{COMPl}_{e} \mathrm{ib}$ examples, we used a modified matrix $E$, $E=\operatorname{diag}\left(I_{n-1}, 0\right)$. With this modification, 24 systems (AC9, HE2, HE3, JE2, DIS4, BDT2, PAS, NN1, NN2, NN9, NN12, NN15, NN16, FS, ROC1-ROC10) became improper, and therefore, these systems have infinite values for the $\mathscr{L}_{\infty}$-norm and corresponding peak frequencies.

Infinite $\mathscr{L}_{\infty}$-norm was also obtained for 14 modified systems with poles on the imaginary axis, usually, in the origin (AC1, AC2, AC11, JE3, REA3, IH, CSE1, CSE2, TF1TF 3, NN6, NN7, and TMD). Actually, the examples CSE1 and CSE2 have an eigenvalue at about $-6.42 \cdot 10^{-17}$, and $2.14 \cdot 10^{-16}$, respectively, as in the standard case. Again, the MATLAB function norm reported $\mathscr{L}_{\infty}$-norms of the order $10^{16}$ for CSE 1 and CSE2.

Notable differences between the results returned by the two solvers have been found for the modified examples EB3-EB6. Both MATLAB norm and the initial version of the SLICOT solver gave infinite norms for the examples EB4-EB6. Actually, norm considered that EB3-EB6 are improper, and returned an infinite peak frequency, while the SLICOT solver returned a finite one for each of these examples. On the other hand, both solvers found finite $\mathscr{L}_{\infty}$ norms for the examples EB1 and EB2. This difference in behaviour is rather strange, since all examples EB1-EB6 
have the same structure, only the order and some of the entries of $B$ (and $C=B^{T}$ ), and of the diagonal of $A$ differ. A deeper investigation revealed that all these systems are proper, and the $\mathscr{L}_{\infty}$-norm is finite. For instance, Fig. 2 shows the singular value plot for the modified example EB3. Moreover, the singular values have been computed for increasing frequencies, and they tend to a finite value, not to infinity. Similar graphs are obtained for $\mathrm{EB} 4-\mathrm{EB} 6$, but there are more peaks. Specifically, there are $(n-2) / 2$ peaks, i.e., 9 for $\mathrm{EB} 4,19$ for $\mathrm{EB} 5,79$ for $\mathrm{EB} 6$.

The singular values for the modified example EB4 have been computed for frequencies $10^{k}$, with $k=1$ : 16 and $k=20: 20: 300$. The singular values tend to $4.9999997110265707 \cdot 10^{4}$. The singular values for EB5 and EB 6 tend to smaller values, of order of $10^{-3}$.

The modified systems EB1-EB6 have poles close to the imaginary axis. For instance, the modified system EB 4 has the poles $\lambda_{i}, i=1: 20(n=20)$,

$$
-10^{-7} \pm \mathrm{i},-4 \cdot 10^{-7} \pm 4 \mathrm{i}, \ldots,-8.1 \cdot 10^{-6} \pm 81 \mathrm{i},-5 \cdot 10^{8}, \infty \text {. }
$$

When looking for poles on (or close to) the imaginary axis, the solvers compare the smallest absolute real part, $r_{\min }$, with the largest finite magnitude, $\left|\lambda_{\max }\right|$. If $r_{\min }<\left(c+\left|\lambda_{\max }\right|\right) \varepsilon$, where $c$ is a constant, then it is decided that there are poles on the imaginary axis. A usual value for $c$ is 1000 , and with this value, the test is satisfied. If the bound above is slightly modified and the solver continues the calculations, the $\mathscr{L}_{\infty}$ norm is found as $4.877604143334732 \cdot 10^{6}$, for the peak frequency 0.99999999999999899. These values are returned by the latest version of the SLICOT solver. Similar comments apply for EB5 and EB6.

The $\mathscr{L}_{\infty}$-norms computed by the two solvers for all other examples have been compared. The SLICOT solver found a slightly larger norm than the MATLAB function norm for 57 examples, and an equal norm for 21 examples. We used again the Euclidean norm of the vector of all finite $\mathscr{L}_{\infty}$ norms in a global measure. The relative difference of the two Euclidean norms corresponding to the two solvers was $2.28 \cdot 10^{-6}$, which shows a very good agreement. However, the difference in the execution time was important, but not so large as in the standard case. The mean value of the ratios of the CPU times for the MATLAB solver and the SLICOT solver for all 152 examples was about 20, and the minimum value was about 1.02 (for example TL). For the descriptor case, both solvers operated on pencils. To show the speed improvement, Fig. 3 plots the speed-up factors for the 12 larger problems in the HF2D group (with orders between 484 and 576). The speed-up factor is over 2.4, which is impressive. (However, this is small in comparison to the speed-up factor in the standard case, when it was 4.8.) This factor was higher for the smaller HF 2D examples (with orders less than 484). The speed-up factor for the examples NN18, CM5, CM6, CM5_IS, and CM6_IS varies between 2.11 and 2.45 . These five examples account for about $61 \%$ of the total CPU time.

The number of iterations and the number of test frequencies used at each iteration have been recorded for both

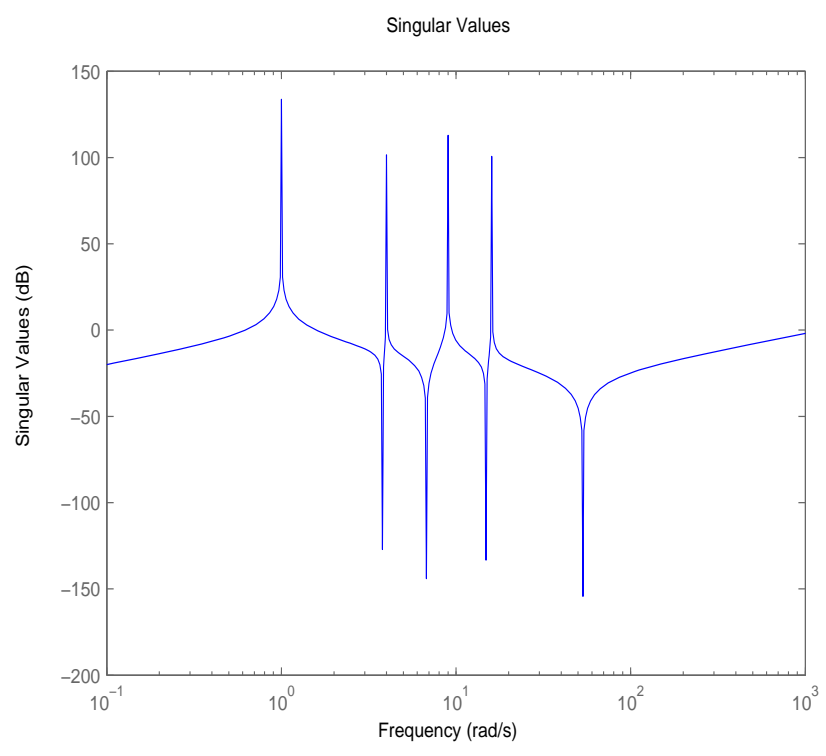

Fig. 2. Singular value plot for the modified example EB3.

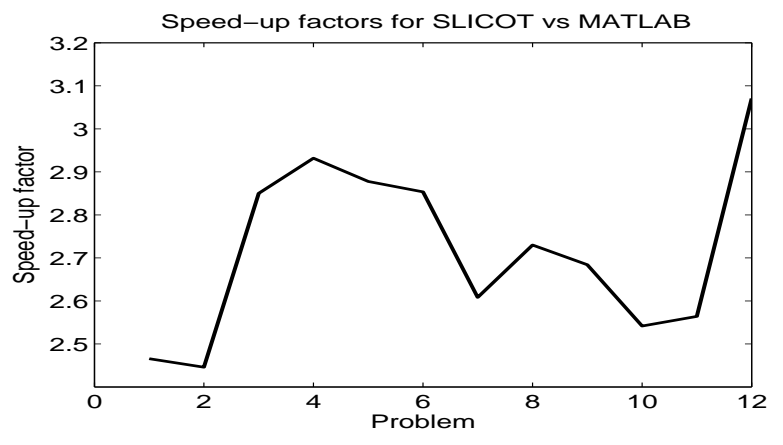

Fig. 3. The ratios between the CPU times needed by the MATLAB norm and SLICOT structure preserving solver for modified $\mathrm{COMPl}_{e}$ ib HF2D larger examples.

solvers, and they agreed very well, with few exceptions. The cases where they differ are reported in Table II. The numbers of test frequencies for each example are their sum for all iterations.

The initial test frequencies (before starting the iterative process) are not counted. Their number differ between the two solvers for 9 examples, and are usually smaller for the MATLAB solver, since it starts by simplifying the system realization. Despite these differences, the $\mathscr{L}_{\infty}$-norms computed by the two solvers agreed very well. Usually, a larger number of iterations or of test frequencies led to a slightly higher $\mathscr{L}_{\infty}$-norm.

Fig. 4 shows the bar graph of the number of iterations needed by the SLICOT $\mathscr{L}_{\infty}$-norm solver versus the number of modified $\mathrm{COMPl}_{e} \mathrm{ib}$ examples. The number of examples requiring $0,1, \ldots, 4$ iterations was $38,68,22,17$, and 7 , respectively. The first number corresponds to the examples with infinite $\mathscr{L}_{\infty}$-norm.

The number of test frequencies was almost always 2 in 
TABLE II

NUMBER OF ITERATIONS AND NUMBER OF TEST FREQUENCIES, WHEN DIFFERENT FOR SLICOT AND MATLAB SOLVERS, APPLIED TO MODIFIED COMPL $\mathrm{L}_{e}$ IB EXAMPLES

\begin{tabular}{|l||c|c|c|c|}
\hline \multicolumn{1}{|l||}{ Examples } & \multicolumn{2}{c|}{ Number of iterations } & \multicolumn{2}{c|}{ Number of frequencies } \\
\cline { 2 - 5 } & SLICOT & MATLAB & SLICOT & MATLAB \\
\hline HE6, HE7 & 3 & 2 & 4 & 2 \\
BDT1 & 1 & 1 & 1 & 0 \\
EB3 & 1 & 0 & 4 & 0 \\
TL & 4 & 1 & 19 & 0 \\
CDP & 2 & 1 & 1 & 0 \\
CM1, CM2, CM3 & 1 & 1 & 1 & 0 \\
CM4 & 1 & 1 & 2 & 0 \\
CM5 & 1 & 1 & 1 & 0 \\
CM1_IS, CM2_IS & 2 & 2 & 3 & 4 \\
CM4_IS-CM6_IS & 2 & 2 & 3 & 4 \\
CM3_IS & 2 & 1 & 3 & 0 \\
DLR2, DLR3 & 1 & 1 & 1 & 0 \\
ISS1, ISS2 & 2 & 2 & 3 & 2 \\
\hline
\end{tabular}

Number of iterations of SLICOT $L_{\infty}-$ norm solver

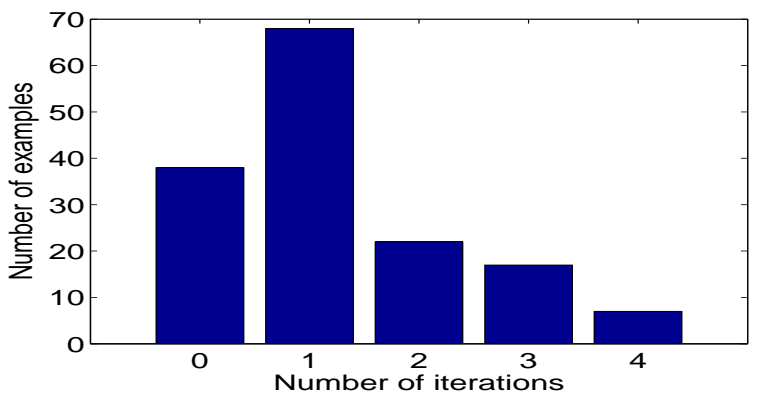

Fig. 4. Bar graph of the number of iterations versus the number of modified examples from the $\mathrm{COMPl}_{e}$ ib collection.

each iteration, except for the last, when it was 0 for all examples, except BDT1, CM1-CM3, CM5, CM1_IS-CM6_IS, DLR2, DLR3, ISS1, and ISS2 with one test frequency, CM4 and $\mathrm{CM} 6$ with 2, and EB3 with 4 test frequencies. For the TL example, the SLICOT solver used 2, 7, 10, and 0 test frequencies in the four iterations.

This investigation reveals that the greater efficiency of the new SLICOT solver is due to the use of structure preserving algorithms. No essential differences in the number of iterations, or of test frequencies at each iteration, have been found.

\section{ACKNOWLEDGMENT}

The authors thank Matthias Voigt who wrote the preliminary versions of some routines, and the reviewers for their constructive comments.

\section{REFERENCES}

[1] P. Benner, V. Mehrmann, and H. Xu, "A new method for computing the stable invariant subspace of a real Hamiltonian matrix," J. Comput. Appl. Math., vol. 86, pp. 17-43, Nov. 1997.

[2] _ "A numerically stable, structure preserving method for computing the eigenvalues of real Hamiltonian or symplectic pencils," Numer. Math., vol. 78, no. 3, pp. 329-358, Jan. 1998.

[3] P. Benner, R. Byers, V. Mehrmann, and H. Xu, "Numerical computation of deflating subspaces of skew Hamiltonian/Hamiltonian pencils," SIAM J. Matrix Anal. Appl., vol. 24, no. 1, pp. 165-190, 2002.
[4] P. Benner, R. Byers, P. Losse, V. Mehrmann, and H. Xu, "Numerical solution of real skew-Hamiltonian/Hamiltonian eigenproblems," Technische Universität Chemnitz, Chemnitz, Tech. Rep., Nov. 2007.

[5] P. Benner, V. Mehrmann, V. Sima, S. Van Huffel, and A. Varga, "SLICOT - A subroutine library in systems and control theory," in Applied and Computational Control, Signals, and Circuits, B. N. Datta, Ed. Boston, MA: Birkhäuser, 1999, vol. 1, chapter 10, pp. 499 539.

[6] V. Sima, "SLICOT-based advanced automatic control computations," in Advances in Automatic Control, M. Voicu, Ed. Boston, MA: Kluwer Academic Publishers, 2004, pp. 337-349.

[7] S. Van Huffel, V. Sima, A. Varga, S. Hammarling, and F. Delebecque, "High-performance numerical software for control," IEEE Control Syst. Mag., vol. 24, no. 1, pp. 60-76, Feb. 2004.

[8] P. Benner, D. Kressner, V. Sima, and A. Varga, "Die SLICOTToolboxen für Matlab," at-Automatisierungstechnik, vol. 58, no. 1, pp. 15-25, Jan. 2010

[9] V. Sima, "Structure-preserving computation of stable deflating subspaces," in Proc. of the 10th IFAC Workshop "Adaptation and Learning in Control and Signal Processing”, Antalya, Turkey, 26-28 August 2010. IFAC-PapersOnLine, Volume 10, Part 1, 2010.

[10] — "Computational experience with structure-preserving Hamiltonian solvers in complex spaces," in Proc. of the PhysCon 2011 - 5th International Scientific Conf. on Physics and Control, León, Spain, September 5-8, 2011. IPACS Electronic library.

[11] _ - "Performance of skew-Hamiltonian/Hamiltonian solvers for automatic control computations," in Proc. of VI-th International Conf “ASTR 2011 Academic Days". Timişoara, September 22-23, 2011, Part I. Editura AGIR, 2011, pp. 130-137.

[12] P. Benner, V. Sima, and M. Voigt, "Robust and efficient algorithms for $\mathscr{L}_{\infty}$-norm computations for descriptor systems," in 7th IFAC Symp. on Robust Control Design, 2012. To appear.

[13] V. Sima, "Efficient algorithm for $L_{\infty}$-norm calculations," in Proc. of the 5th IFAC Symposium on Robust Control Design, July 5-7, 2006, Toulouse, France. Curran Associates, Inc., 2006.

[14] P. Benner, R. Byers, and E. Barth, "Algorithm 800: Fortran 77 subroutines for computing the eigenvalues of Hamiltonian matrices I: The square reduced method," ACM Trans. Math. Softw., vol. 26, nr. 1, pp. 49-77, Mar. 2000.

[15] P. Benner, V. Sima, and M. Voigt, " $\mathscr{L}_{\infty}$-norm computation for continuous-time descriptor systems using structured matrix pencils," IEEE Trans. Automat. Contr., vol. AC-57, no. 1, pp. 233-238, Jan 2012.

[16] "Control System Toolbox User's Guide. Version 9," The MathWorks, Inc., 3 Apple Hill Drive, Natick, MA, 01760-2098, 2011.

[17] F. Leibfritz and W. Lipinski, " $C O M P l_{e} i b 1.0$ - User manual and quick reference,” Department of Mathematics, University of Trier, D-54286 Trier, Germany, Tech. Rep., 2004

[18] A. W. Bojanczyk, G. Golub, and P. Van Dooren, "The periodic Schur decomposition: algorithms and applications," in Proc. of the SPIE Conf. Advanced Signal Processing Algorithms, Architectures, and Implementations III, vol. 1770, pp. 31-42, Nov. 1992.

[19] J. Sreedhar and P. Van Dooren, "Periodic Schur form and some matrix equations," in Systems and Networks: Mathematical Theory and Applications, Proc. of the Symposium on the Mathematical Theory of Networks and Systems (MTNS'93), Regensburg, Germany, 2-6 August 1993, vol. 1. John Wiley \& Sons, pp. 339-362, 1994.

[20] R. Granat, B. Kågström, and D. Kressner, "Computing periodic deflating subspaces associated with a specified set of eigenvalues,' BIT Numerical Mathematics, vol. 47, nr. 4, pp. 763-791, Dec. 2007.

[21] _ "MATLAB tools for solving periodic eigenvalue problems," in Third IFAC Workshop on Periodic Control Systems, August, 29-31, 2007, Saint Petersburg, Russia.

[22] S. Boyd, V. Balakrishnan, and P. Kabamba, "A bisection method for computing the $H_{\infty}$ norm of a transfer matrix and related problems," Mathematics of Control, Signals, and Systems, vol. 2, no. 3, pp. 207 219, Sept. 1989.

[23] N. A. Bruinsma and M. Steinbuch, "A fast algorithm to compute the $H_{\infty}$-norm of a transfer function," Systems Control Lett., vol. 14, no. 4 , pp. 287-293, Apr. 1990 .

[24] V. Sima, "Computational experience with structure-preserving Hamiltonian solvers in optimal control," in Proc. of the "8th International Conf. on Informatics in Control, Automation and Robotics", Noordwijkerhout, The Netherlands, 28-31 July, 2011, vol. 1, pp. 91-96. 